\title{
Loi de comportement en plasticité cristalline pour acier à basse température
}

\author{
Ghiath Monnet ${ }^{1}$ et Ludovic Vincent ${ }^{2, a}$ \\ 1 EDF R\&D, MMC, Avenue des Renardières, Écuelles, 77818 Moret-sur-Loing Cedex, France \\ 2 CEA Saclay, DEN, SRMA, 91191 Gif-sur-Yvette Cedex, France
}

Reçu le 28 avril 2011, accepté le 11 mai 2011

\begin{abstract}
Résumé - Dans le cadre de la prévision par une approche multiéchelles de l'évolution de la courbe de transition ductile/fragile des aciers de cuves des centrales nucléaires en fonction de l'irradiation, on commence par tenter de reproduire le comportement mécanique à basse température de ces matériaux à l'échelle de l'agrégat. Partant d'une analyse des résultats numériques obtenus par dynamique des dislocations discrètes dans ce régime de température, une nouvelle loi de comportement en plasticité cristalline est donc proposée avec un minimum de paramètres ajustables sur résultats macroscopiques.
\end{abstract}

Mots clés : Plasticité cristalline / dislocations / matériaux cubiques centrés / activation thermique

Abstract - Crytalline plasticity constitutive equations for BCC steel at low temperature. The prediction of the irradiation-induced evolution of the ductile-fragile transition curve of pressure vessel steels is a major research topic in the nuclear industry. Multiscale approaches starting from ab initio scale up to macroscopic continuum mechanics are currently investigated through the European project PERFORM60. At the intermediate level of cristal plasticity, several effects need to be described accurately before considering the introduction of irradiation hardening mechanisms, such as the thermal activity of dislocations slip, the different mobilities between screw and edge dislocations at low temperature. These effects should be introduced in a cristal plasticity law used in finite-element simulations of polycrystalline aggregates. Accordingly, a new cristal plasticity law is proposed in this paper based on a critical analysis of previous numerical results obtained with a discrete dislocations dynamics code.

Key words: Cristal plasticity / dislocations / body-centered cubic materials / thermal activity

\section{Introduction}

Dans un contexte de prolongation de la durée de vie des centrales nucléaires existantes, on cherche à prévoir l'évolution sur le long terme de la fragilisation due à l'irradiation neutronique de l'acier constituant les cuves des réacteurs, un acier bainitique 16MND5 de structure cristallographique cubique centrée. Cette fragilisation se caractérise macroscopiquement par un décalage vers les hautes températures de la transition ductile-fragile de la courbe de ténacité. Ce décalage de transition s'explique en partie par le durcissement du matériau provoqué par l'introduction de défauts d'irradiation (cavités, boucles lacunaires ou interstitielles) qui s'opposent au glissement des dislocations. Les dommages d'irradiation produits à une échelle atomique ont ainsi des conséquences à l'échelle de la structure et une approche multiéchelles a ici tout son

${ }^{a}$ Auteur pour correspondance : ludovic.vincent@cea.fr sens. Cette démarche est soutenue par un projet européen PERFORM60 [http://www.perform60.net] lequel implique un grand nombre de laboratoires de recherche.

Pour prévoir l'évolution du comportement mécanique des aciers de cuve en présence de défauts d'irradiation sur une large plage de température, il convient au préalable d'être capable de bien représenter le comportement du matériau à l'état non-irradié sur la même plage de température.

Cet objectif est ici poursuivi à l'échelle de la plasticité cristalline qui permet de remonter des informations sur le comportement mécanique d'un grain à l'échelle de celui d'un élément de volume des composants, l'élément de volume étant constitué d'un agrégat de grains. Plusieurs outils d'homogénéisation sont disponibles pour faire ce changement d'échelles, et on choisit ici un code de calcul aux éléments-finis qui permet de prendre en compte explicitement les effets de voisinage entre grains. Le comportement mécanique de chaque grain est décrit dans ce 


\section{Nomenclature}

\begin{tabular}{|c|c|}
\hline$\alpha$ & constante intervenant dans le calcul de la longueur de segment vis \\
\hline$\alpha_{\text {auto }}$ & constante intervenant dans le calcul de cission d'auto écrouissage \\
\hline$a^{\mathrm{su}}$ & paramètres d'interactions entre systèmes de glissement \\
\hline$b$ & norme du vecteur de Burgers ( $\mathrm{mm})$ \\
\hline$\underline{\underline{\underline{\underline{C}}}}$ & tenseur d'ordre 4 des modules (MPa) \\
\hline$\Delta G_{0}$ & énergie d'activation $(\mathrm{eV})$ \\
\hline$\underline{\underline{E}}^{e}$ & tenseur d'ordre 2 déformation élastique de Green-Lagrange \\
\hline$\underline{\underline{F}}$ & tenseur d'ordre 2 gradient de la transformation \\
\hline$\underline{\underline{F}}^{\mathrm{e}}$ & tenseur d'ordre 2 partie élastique du gradient de la transformation \\
\hline$\underline{F}^{\mathrm{p}}$ & tenseur d'ordre 2 partie plastique du gradient de la transformation \\
\hline$\dot{\gamma}$ & taux de glissement \\
\hline$g_{\mathrm{c}}$ & distance d'annihilation $(\mathrm{mm})$ \\
\hline$H$ & taux d'activation $\left(\mathrm{s}^{-1}\right)$ \\
\hline$k$ & constante de Boltzmann $\left(\mathrm{eV} \cdot \mathrm{K}^{-1}\right)$ \\
\hline$K$ & constante intervenant dans l'estimation du libre parcours moyen \\
\hline$l_{\mathrm{c}}$ & longueur critique d'un double décrochement (mm) \\
\hline$l_{\mathrm{sc}}$ & longueur de segment vis $(\mathrm{mm})$ \\
\hline$L$ & espacement moyen entre obstacles pour une dislocation $(\mathrm{mm})$ \\
\hline$\mu$ & module de cisaillement (MPa) \\
\hline$\underline{m}$ & vecteur direction de glissement d'un système de glissement \\
\hline$\underline{n}$ & vecteur normale au plan d'un système de glissement \\
\hline$\underline{\underline{N}}$ & tenseur d'ordre 2 de Schmid définissant l'orientation d'un système de glissement \\
\hline$R$ & rayon de courbure de dislocation $(\mathrm{mm})$ \\
\hline$\rho$ & densité de dislocations $\left(\mathrm{mm}^{-2}\right)$ \\
\hline$T$ & température absolue $(\mathrm{K})$ \\
\hline$\tau_{\text {app }}$ & cission appliquée sur le système de glissement $(\mathrm{MPa})$ \\
\hline$\tau_{\text {eff }}$ & cission effective appliquée sur le système de glissement (MPa) \\
\hline$\tau_{\mathrm{F}}$ & cission de friction de réseau $(\mathrm{MPa})$ \\
\hline$\tau_{\mathrm{lt}}$ & tension de ligne $(\mathrm{MPa})$ \\
\hline$\tau_{0}$ & cission critique intervenant dans la loi d'écoulement thermique activée ( $\mathrm{MPa})$ \\
\hline$\tau_{\text {self }}$ & cission d'autoécrouissage (MPa) \\
\hline$v_{\mathrm{sc}}$ & vitesse d'un segment de dislocation vis $\left(\mathrm{mm} \cdot \mathrm{s}^{-1}\right)$ \\
\hline
\end{tabular}

type de simulations à l'aide d'une loi de comportement autrement appelée loi de plasticité cristalline. Cette loi représente en fait le mouvement collectif des dislocations qui interagissent au sein d'un grain.

Les simulations de dynamique des dislocations (DD) ont justement pour ambition de représenter ce mouvement collectif des dislocations moyennant un choix fait sur la loi de mobilité des dislocations qui s'appuie sur une analyse fine de résultats expérimentaux et de simulations en dynamique moléculaire.

On propose dans cet article de tirer les enseignements de résultats de simulations numériques en DD $[1,2]$ pour écrire une nouvelle loi de plasticité cristalline pour matériaux cubique centré (BCC), dans le régime thermiquement activé. Le jeu d'équations constitutives de cette loi est présenté avant de l'appliquer au cas simple d'une traction sur une poutre monocristalline.

\section{Loi de plasticité cristalline}

Des observations expérimentales [3] et de récents travaux en $\mathrm{DD}$ sur le comportement à basse température du fer $[1,2]$ ont mis en évidence des propriétés importantes de la déformation plastique :

- La contrainte d'écoulement $\tau_{\text {app }}$ est indépendante de la mobilité des dislocations coins.

- $\grave{A}$ vitesse constante $v_{\text {sc }}$, la cission effective sur un segment rectiligne de dislocation vis $\tau_{\text {eff }}$ dépend de la longueur de ce segment $l_{\mathrm{sc}}$.

- Dans un régime stationnaire, la partie non-vis des dislocations est courbée et le rayon de courbure vaut $R=\mu b / \tau_{\text {eff }}$, où $\mu$ est le module de cisaillement et $b$ la norme du vecteur de Burgers.

- Lorsque la longueur de segment vis tend vers zéro, la dislocation vis se courbe et $\tau_{\text {eff }}$ augmente pour compenser la tension de ligne $\tau_{\mathrm{lt}}$.

Partant de ces remarques principales, il est possible de proposer des relations de comportement en plasticité cristalline. Tout d'abord, la relation d'Orowan reliant la vitesse des dislocations mobiles au taux de glissement sur un système $s$ fournit :

$$
\dot{\gamma}^{\mathrm{s}}=4 b \rho_{\mathrm{sc}}^{\mathrm{s}} H l_{\mathrm{sc}}^{\mathrm{s}} \exp \left(-\frac{\Delta G_{0}}{k T}\right) \sinh \left(\frac{\Delta G_{0}}{k T} \sqrt{\frac{\tau_{\mathrm{eff}}^{\mathrm{s}}}{\tau_{o}}}\right)
$$


Tableau 1. Valeurs des paramètres dépendant du matériau.

\begin{tabular}{ccccccc}
\hline$E(\mathrm{GPa})$ & $\nu$ & $b(\mathrm{~nm})$ & $H\left(\mathrm{~s}^{-1}\right)$ & $\Delta G_{0}(\mathrm{eV})$ & $\tau_{0}(\mathrm{MPa})$ & $\rho_{\text {ini }}^{\mathrm{s}}\left(\mathrm{mm}^{-2}\right)$ \\
\hline $225,91-44 \mathrm{~T}$ & 0,29 & 0,248 & $1,5 \times 10^{11}$ & 0,84 & 363 & $10^{6}$ \\
$l_{\mathrm{c}}(\mathrm{nm})$ & $\tau_{\mathrm{F}}(\mathrm{MPa})$ & $g_{\mathrm{c}}(\mathrm{nm})$ & $a^{\text {su }}$ & $\alpha_{\text {auto }}$ & $\alpha$ & $K$ \\
4,96 & 10 & 4 & 0,1225 & 0,25 & 0,35 & 10 \\
\hline
\end{tabular}

Les indices haut font référence au système de glissement considéré alors que les indices bas précisent de quel type de dislocation il s'agit (sc : screw/vis, ed : edge/coin). Les paramètres $H, \Delta G_{0}$ et $\tau_{0}$ sont identifiés à partir de résultats expérimentaux [1]. $k$ est la constante de Boltzmann et $T$ la température absolue. $\rho_{\mathrm{sc}}^{\mathrm{s}}$ représente la densité de dislocations vis du système $s$, considérée égale à la densité de dislocation totale du système $\rho^{\mathrm{s}}$ par simplicité dont l'évolution est décrite par l'équation différentielle suivante [4] :

$$
\dot{\rho}^{\mathrm{s}}=\frac{\left|\dot{\gamma}^{\mathrm{s}}\right|}{b}\left(\frac{\alpha \sqrt{\sum_{u \neq \mathrm{s}} \rho^{u}}}{K}-g_{\mathrm{c}} \rho^{\mathrm{s}}\right)
$$

avec $\alpha, K$ et $g_{\mathrm{c}}$ trois constantes dépendantes du matériau.

La longueur de segment vis $l_{\mathrm{sc}}^{\mathrm{s}}$ est calculée comme une fonction de l'espacement moyen entre obstacles $L^{\mathrm{s}}: l_{\mathrm{sc}}^{\mathrm{s}}=$ $\max \left(l_{\mathrm{c}} ; L^{\mathrm{s}}-2 \alpha R^{\mathrm{s}}\right)$ où $l_{\mathrm{c}}$ est la longueur critique d'un double décrochement représentant la limite inférieure de $l_{\mathrm{sc}}^{\mathrm{s}}$. La distance moyenne entre obstacles s'écrit :

$$
L^{\mathrm{s}}=\frac{1}{\sqrt{\sum_{u \neq \mathrm{s}} a^{\mathrm{su}} \rho^{u}}}
$$

avec $a^{\text {su }}$ les paramètres d'interaction que l'on peut calculer à l'aide de simulations DD. Le rayon de courbure $R^{\mathrm{s}}$ peut quant à lui s'écrire :

$$
R^{\mathrm{s}}=\frac{\mu b}{\left|\tau_{\mathrm{appl}}^{\mathrm{s}}\right|-\tau_{\mathrm{self}}^{\mathrm{s}}-\tau_{\mathrm{F}}}
$$

avec $\tau_{\mathrm{F}}$ un terme de friction de réseau et $\tau_{\text {self }}^{\mathrm{s}}$ un terme d'auto écrouissage qui s'écrit : $\tau_{\text {self }}^{\mathrm{s}}=\alpha_{\text {auto }} \mu b \sqrt{\rho^{\mathrm{s}}}$.

La cission effective s'écrit enfin :

$$
\tau_{\text {eff }}^{\mathrm{s}}=\left|\tau_{\text {app }}^{\mathrm{s}}\right|-\left(\tau_{\text {self }}^{\mathrm{s}}+\tau_{\mathrm{LT}}^{\mathrm{s}}+\tau_{\mathrm{F}}\right)
$$

avec $\tau_{\text {LT }}^{\mathrm{s}}$ un terme de tension de ligne. Cette contribution reste nulle tant que la longueur de segment vis $l_{\mathrm{sc}}^{\mathrm{s}}$ reste supérieure à la longueur critique $l_{\mathrm{c}}$. Lorsque la température augmente ou que le niveau de déformation conduit à bloquer $l_{\mathrm{sc}}^{\mathrm{s}}$ à sa plus faible valeur, la tension de ligne s'écrit alors :

$$
\tau_{\mathrm{LT}}^{\mathrm{s}}=\mu b\left(\frac{1}{L^{\mathrm{s}}}-\frac{1}{2 \alpha R^{\mathrm{s}}+l_{\mathrm{c}}}\right)
$$

Les paramètres de la loi de plasticité cristalline dépendant du matériau sont reportés dans le tableau 1.

\section{Implantation numérique}

La loi de plasticité cristalline présentée dans la section précédente est introduite dans le code de calcul aux éléments-finis CAST3M (http://www-cast3m.cea. $\mathrm{fr} /$ ). La résolution numérique des équations différentielles est réalisée à l'aide d'une procédure Runge-Kutta d'intégration explicite à pas de temps adaptatif en fonction d'un critère d'erreur [5].

Pour pouvoir prendre en compte les rotations du réseau cristallin au cours de la déformation plastique, les relations de comportement sont au préalable introduites dans un cadre de grandes déformations [6]. On utilise la décomposition classique [7] du gradient de la transformation $\underline{\underline{F}}$ en sa partie plastique $\underline{\underline{F}}^{\mathrm{p}}$ et élastique $\underline{\underline{F}}^{\mathrm{e}}$.

$$
\underline{\underline{F}}=\underline{\underline{F}}^{\mathrm{e}} \underline{\underline{F}}^{\mathrm{p}}
$$

Dans ce cadre, la cission résolue sur chaque système de glissement s'écrit [6] :

$$
\tau_{\text {app }}^{\mathrm{s}}=\left(\underline{\underline{F}}^{\mathrm{e}^{\mathrm{T}}} \underline{\underline{\underline{F}}} \underline{\underline{\underline{ }}}: \underline{\underline{E^{\mathrm{e}}}}\right): \underline{\underline{N}}^{\mathrm{s}}
$$

avec $\underline{\underline{\underline{C}}}$ le tenseur des modules, $\underline{\underline{E}}^{\mathrm{e}}$ la déformation élastique de Green-Lagrange et $\underline{\underline{N}}^{\mathrm{s}}$ le tenseur de Schmid défini par $\underline{\underline{N}}^{\mathrm{s}}=\underline{m}^{s} \otimes \underline{n}^{\mathrm{s}}$, avec $\underline{m}^{\mathrm{s}}$ la direction de glissement et $\underline{n}^{\mathrm{s}}$ la normale au plan de glissement. On prend l'hypothèse simplificatrice d'une élasticité isotrope définie par le module de Young $E$ du matériau et le coefficient de Poisson $\nu$. Dans ce cas, le module de cisaillement s'écrit $\mu=\frac{E}{2(1+\nu)}$. La dépendance en température du comportement élastique est prise uniquement dans la valeur du module d'Young (i.e. $\nu$ constant).

À chaque pas de temps, le code de calcul fournit la nouvelle valeur de $\underline{\underline{F}}$ et connaissant $\underline{\underline{F}}^{\mathrm{p}}$ stocké dans des variables internes, on peut ainsi de proche en proche intégrer le comportement du matériau. En post-traitement, il est également possible de connaître la part de rotation élastique comprise dans $\underline{\underline{F}}$ ou $\underline{\underline{F}}^{\mathrm{e}}$, ce qui lors d'un essai de traction revient à connaître la rotation du réseau cristallin. Pour ce faire, on utilise la décomposition $\underline{\underline{F}}^{\mathrm{e}}=\underline{\underline{R U}}$ avec $\underline{\underline{R}}$ un tenseur orthogonal et $\underline{\underline{U}}$ un tenseur symétrique défini positif [8]. 


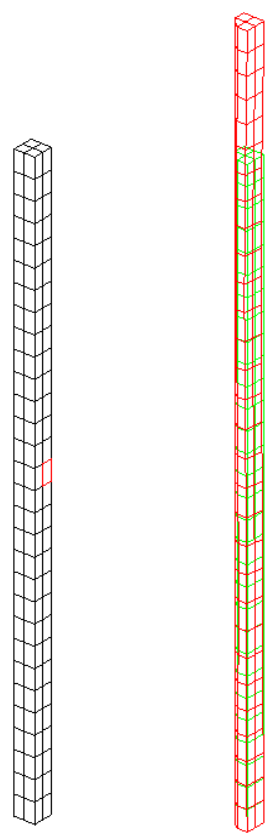

(a)

(b)

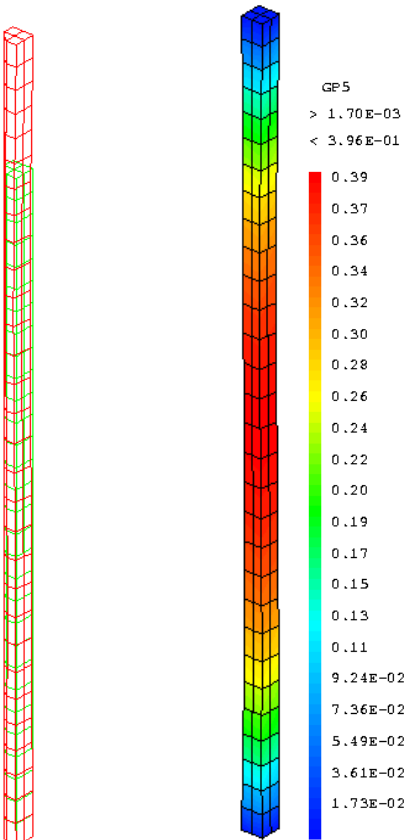

(c)

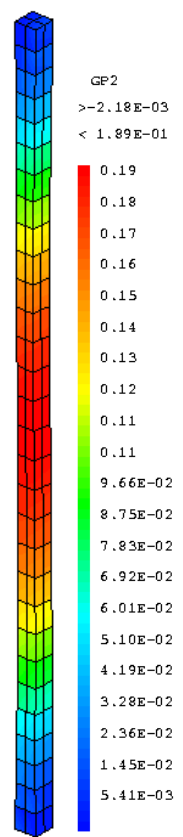

(d)

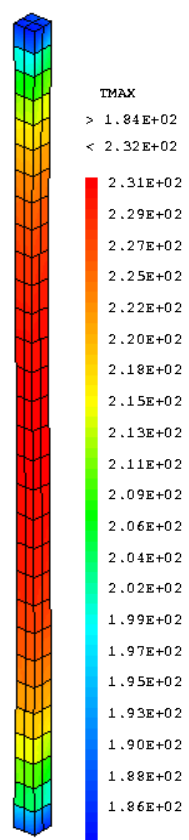

(e)

Fig. 1. Maillage, déformée et champs de principales variables internes d'une poutre monocristalline après $20 \%$ de déformation conventionnelle de traction à 100 K. (a) Maillage. (b) Déformée. (c) $\gamma^{\mathrm{p}}$ Syst. princ. (d) $\gamma^{\mathrm{p}}$ Syst. sec. (e) $\tau^{\mathrm{s}}$ Syst. prim.

\section{Premiers résultats de simulation}

On simule pour différentes températures le comportement en traction d'une poutre monocristalline constituée de $2 \times 2 \times 30$ éléments-finis cubiques à interpolation linéaire du champ de déplacement (CUB8). On oriente le monocristal par rapport à l'axe de traction pour que la déformation ne se fasse initialement que selon un système de glissement. L'axe de traction est ainsi orienté selon la direction $\underline{t}=[-1 ; 5 ; 9]$ dans le repère cristallin.

Pour rendre compte de la rotation du réseau cristallin qui apparaît au cours d'un essai réel de traction sur monocristal après de grands niveaux de déformation, on impose que les faces extrêmes de la poutre restent parallèles et ne se déplacent que dans la direction de traction [9].

Le maillage utilisé ainsi qu'une déformée de l'éprouvette obtenue après $20 \%$ de déformation conventionnelle sont représentés sur la figure 1 . Un exemple de champs de glissement plastique sur le système primaire et secondaire est également représenté sur cette figure ainsi que le champ de cission sur le système principal.

Dans la suite, on représente l'évolution au cours de la déformation des principales variables enregistrées dans un élément fini situé au centre de la poutre (représenté en rouge sur la première image de Fig. 1), et ce pour différentes valeurs de température. Le comportement sur le système primaire est ainsi représenté sur la figure $2 \mathrm{a}$ en fonction de la température. Un point de comparaison intéressant par rapport à des résultats expérimentaux [10-13] et de simulations en DD [1] est l'évolution de la cission critique lors de l'entrée en plasticité en fonction de la température. Cette comparaison est représentée sur la figure $2 \mathrm{~b}$ et l'on constate une bonne reproduction de cette évolution par la loi de plasticité cristalline. Cela n'est en soit pas trop surprenant dans la mesure où la loi d'écoulement (1) s'inspire fortement de la loi de mobilité introduite dans les simulations de DD [1]. Concernant l'écrouissage, on peut constater sur Fig. 2a que celui-ci augmente avec la température (surtout en relatif par rapport à la cission critique), ce qui est en accord avec des résultats expérimentaux de [12].

L'évolution de la longueur de segment vis et de la tension de ligne du système primaire est représenté sur la figure 3. On peut remarquer que la longueur de segment vis est bien une fonction décroissante de la température et de la déformation plastique. Tant que la longueur de segment vis reste supérieure à sa longueur critique $l_{\mathrm{c}}$, la tension de ligne reste nulle, ce qui est observé pour toutes les simulations réalisées à une température inférieure à $250 \mathrm{~K}$ et quel que soit le niveau de déformation.

À partir de $250 \mathrm{~K}$, la longueur de segment vis décroit suffisamment pour atteindre sa valeur minimale, ce qui permet à la tension de ligne d'augmenter en fonction de la déformation plastique. Enfin à $350 \mathrm{~K}$, on constate que la longueur de segment vis est dès le début de la simulation bloquée à sa valeur minimale.

On peut constater sur la figure 4 que l'évolution de la densité de dislocations sur le système primaire et l'activité plastique du système secondaire en fonction de celle du système primaire sont assez peu sensibles à la température simulée.

Enfin, on peut représenter sur la figure 5b l'évolution de la direction de l'axe de traction dans le triangle standard au cours de la simulation, ce qui permet d'évaluer la 


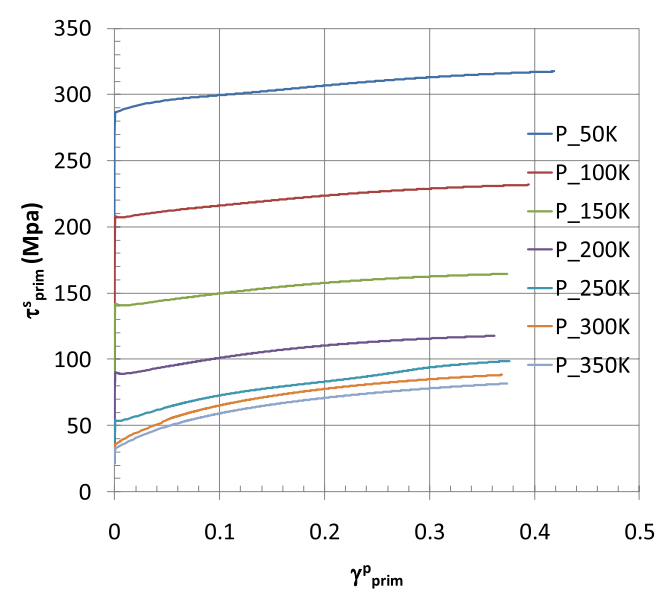

(a)

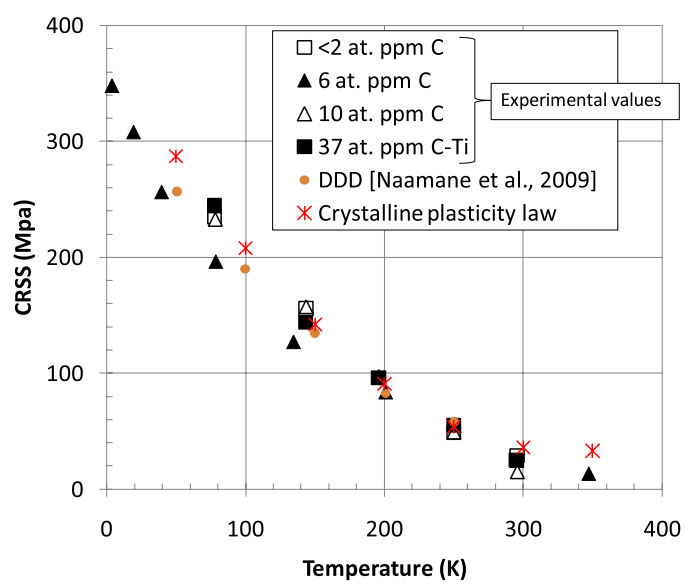

(b)

Fig. 2. Comportement du système principal. Comparaison avec des résultats expérimentaux [10-13] et de DD [1]. (a) Comportement sur le système primaire. (b) Évolution de la cission critique en fonction de la température.

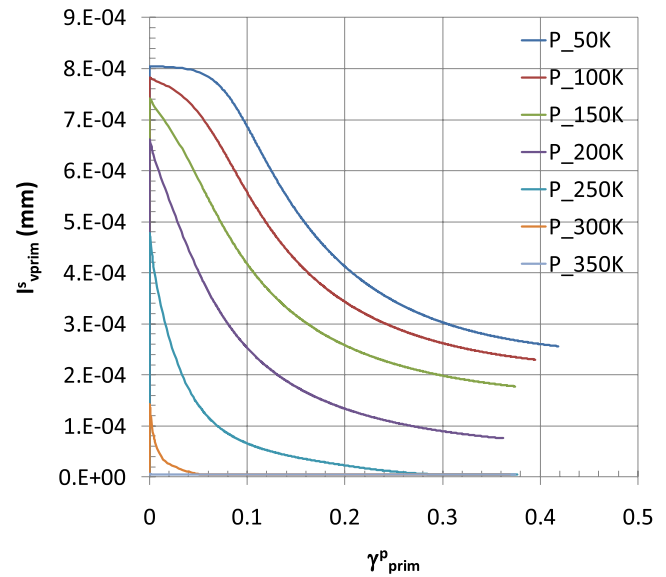

(a)

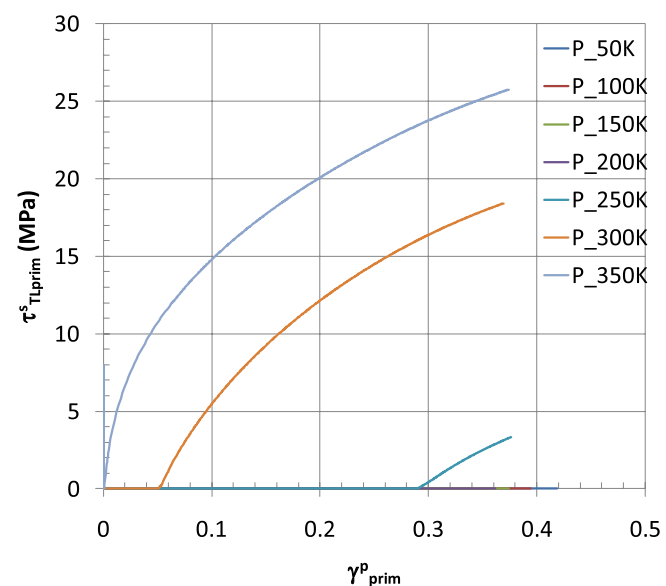

(b)

Fig. 3. Évolution de la longueur de segment vis (a) et de la tension de ligne (b).

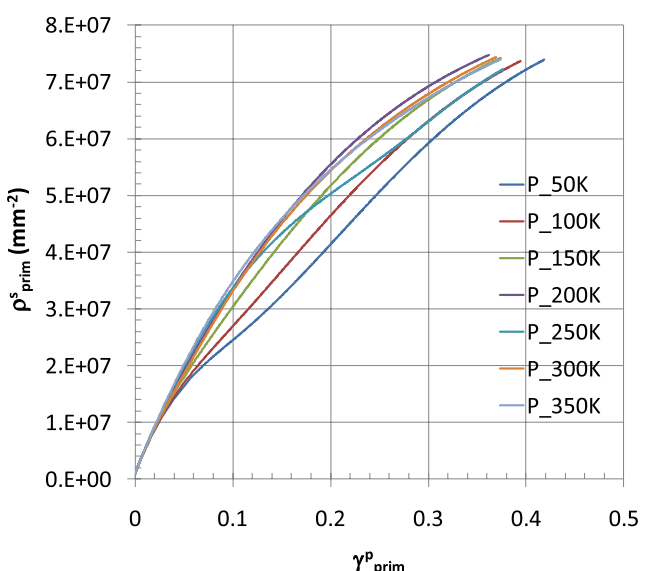

(a)

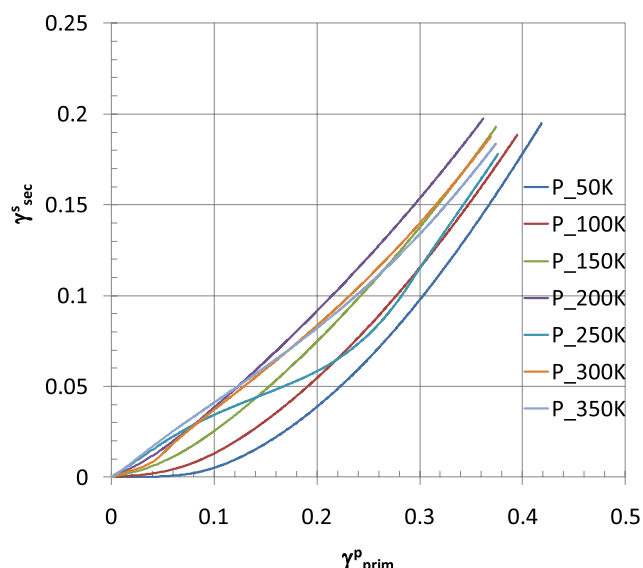

(b)

Fig. 4. (a) Évolution de la densité de dislocation sur le système primaire et (b) évolution du glissement plastique sur le système secondaire en fonction du glissement sur le système primaire. 


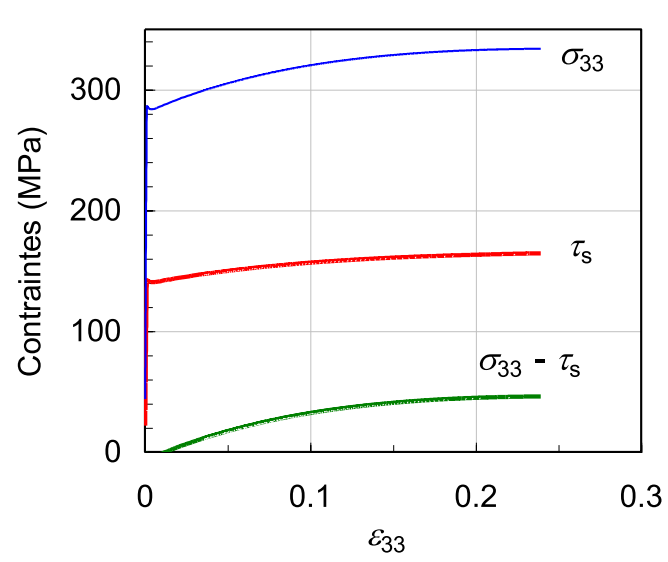

(a)

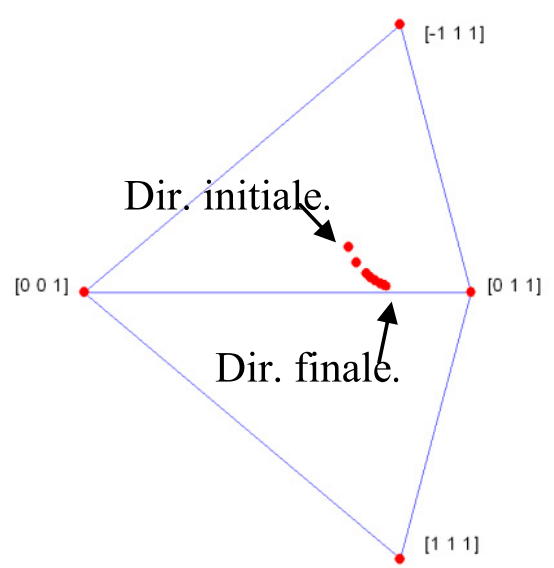

(b)

Fig. 5. (a) Contrainte axiale et résolue sur le système principale et (b) évolution de la direction de traction dans le triangle standard.

rotation du réseau cristallin et de vérifier que celle-ci est bien en accord avec des résultats expérimentaux obtenus à température ambiante $[14,15]$. Du fait de cette rotation, l'augmentation de contrainte axiale macroscopique est plus importante que celle calculée sur le système primaire (Fig. 5a).

\section{Conclusion}

Une loi de plasticité cristalline est proposée pour le régime basse température des matériaux cubique centré en partant de l'analyse de résultats obtenus en dynamique des dislocations. Un premier exemple de simulation sur une poutre monocristalline permet d'évaluer la capacité de cette loi à reproduire certains résultats expérimentaux ou de simulation en DD.

\section{Références}

[1] S. Naamane, G. Monnet, B. Devincre, Low temperature deformation in iron studied with dislocation dynamics simulations, Int. J. Plast. 26 (2010) 84-92

[2] G. Monnet, S. Naamane, B. Devincre, Orowan Strengthening at low temperatures in bcc materials studied by dislocation dynamics simulations, Acta Mater. 59 (2011) 451-461

[3] K. Obrtlik, C.F. Robertson, B. Marini, Dislocation structures in 16MND5 pressure vessel steel strained in uniaxial tension, J. Nucl. Mater. 342 (2005) 35-41

[4] Y. Estrin, H. Mecking, A unified phenomenological description of work-hardening and creep based on oneparameter models, Acta Metall. 32 (1984) 57-70
[5] O. Fandeur, Procédure Runge-Kutta d'intégration explicite à pas de temps adaptatif pour lois de comportement en grandes transformations dans CAST3M, communication personnelle, 2010

[6] J. Besson, G. Cailletaud, J. Chaboche, S. Forest, Mécanique non linéaire des matériaux, Hermès, Paris, 2001

[7] J. Mandel, Plasticité classique et viscoplasticité, CISM Courses and lectures, Udine, Springer Verlag, Berlin, Vol. 97, 1971

[8] S. Forest, M. Amestoy, Mécanique des milieux continus, Cours de l'École des Mines, Les Presses de l'École des Mines, 2008

[9] M. Fivel, S. Forest, Plasticité cristalline et transition d'échelle : cas du monocristal, doc M4-016, Les Techniques de l'Ingénieur

[10] D.J. Quesnel, A. Sato, M. Meshii, Solution softening and hardening in iron-carbon system, Mater. Sci. Eng. 18 (1975) 199-208

[11] W.A. Spitzig, Effects of orientation, temperature and strain rate on deformation of $\mathrm{Fe}-0.16 \mathrm{Wt}$ percent $\mathrm{Ti}$ single-crystals, Mater. Sci. Eng. 12 (1973) 191-202

[12] E. Kuramoto, Y. Aono, K. Kitajima, Thermally activated slip deformation of high-purity iron single-crystals between 4.2-K and 300-K, Scr. Metall. Mater. 13 (1979) 1039-1042

[13] W.A. Spitzig, A.S. Keh, Effect of orientation and temperature on plastic flow properties of iron single crystals, Acta Metall. 18 (1970) 611-622

[14] B. Jaoul, D. Gonzalez, Déformation plastique de monocristaux de fer, J. Mech. Phys. Solids 9 (1961) 16-38

[15] A.S. Keh, Work hardening and deformation sub-structure in iron single crystals deformed in tension at 298 degrees K, Philos. Mag. 12 (1965) 9-30 